emotional lability seen in LE is semiologically distinct from pseudobulbar affect observed in other neurological diseases. While LE is characterised by focal hippocampal atrophy, functional abnormalities in regions interacting with the hippocampus may provide a more parsimonious explanation of emotional lability than the volume of medial temporal lobe structures. Functional abnormalities in parietal regions supporting perspective taking and social-affective processing may compromise patients' emotion regulation.

\section{$33 \quad$ FIRST EPISODE PSYCHOSIS IN A PATIENT WITH EXTENSIVE LEUKOENCEPHALOPATHY DUE TO 3-METHYLGLUTACONIC ACIDURIA TYPE 4}

${ }^{1}$ Rebecca Charles*, ${ }^{2}$ Baskaran Sridharan. ${ }^{1}$ Foundation year 1 Doctor, Combined Healthcare NHS Trust; ${ }^{2}$ Consultant Neuropsychiatrist, Combined Healthcare NHS Trust

\subsection{6/jnnp-2019-BNPA.33}

Objectives/Aims This aim of case report is to discuss the clinical conundrum and diagnostic challenges in a young patient presenting with First Episode Psychosis. Investigations revealed Extensive Leukoencephalopathy due to a rare metabolic disorder- 3-methyloglutaconic aciduria (3-MGA) type IV. Several studies have shown that 3-MGA type IV can present with psychosis, epilepsy or depression as part of the spectrum of symptoms. The role of Organic Brain condition in the onset of first episode psychosis in this patient is discussed in this report.

Methods A 23-year-old female presented with insidious onset of paranoid delusions and auditory hallucinations over an 18 month period on a background of a diagnosis of 3-methylgutaconic aciduria type IV confirmed on urine testing. On admission under Section 2 of the Mental Health Act, she expressed little spontaneous speech and echolalia. She was flat in affect. She appeared vacant in expression, stared inappropriately and was very self-isolating. She was responding to external stimuli. She lacked insight into her condition. Physical examination was unremarkable. An MRI brain scan was performed, and comparison made to scan done previously to demonstrate any interval change and to correlate changes if present to deterioration of clinical symptoms.

Results MRI scan showed extensive diffuse leukoencephalopathy. Comparison to MRI scan done 6 years previously did not show any change or progression to the white matter lesions. An EEG showed a mild degree of general cerebral dysfunction with no interictal epileptiform activity. There was no correlation found between her clinical symptoms of acute onset psychosis and her diagnosis of 3-MGA Type IV. She was commenced on Aripiprazole and her presentation improved significantly. Both Auditory hallucinations and Paranoid delusions improved considerably, with moderate improvement in mood, affect and apathy. Some Catatonic symptoms persisted but were less intense. She was given a diagnosis of Undifferentiated Schizophrenia under ICD 10, as she displayed features of Paranoid, Hebephrenic and Catatonic without clear predominance of particular subtype of Schizophrenia. She was discharged home with follow-up from Neuropsychiatry and community Mental health teams. She continues to be investigated for the genetic cause of 3-methylglucatonic aciduria Type 4.

Conclusions To conclude, although often metabolic disorders, including 3-methylglucaconic aciduria, can present with psychosis, it is prudent to establish a causative link in order to manage appropriately and effectively.

\section{THE FUTURE ROLE OF FMRI NEUROFEEDBACK IN DEPRESSION TREATMENT AND RESEARCH}

*Zahn R, Jaeckle T, Williams SCR, Barker G, Young AH, Basilio R, Moll J. Centre for Affective Disorders, Department of Psychological Medicine Institute of Psychiatry, Psychology and Neuroscience (IoPPN), King's College London

\subsection{6/jnnp-2019-BNPA.34}

Objectives/Aims FMRI-neurofeedback for major depressive disorder (MDD) is of great interest to clinicians and neuroscientists. Here, the aim was to review the current clinical trials evidence.

Methods We undertook a systematic literature search of fMRIneurofeedback trials in MDD, including our unpublished results.

Results fMRI-neurofeedback was effective in current MDD when reinforcing brain responses to positive pictures, ${ }^{1}$ but was not superior to a control neurofeedback intervention in a recent randomised controlled trial ${ }^{2}$ (RCT). Another RCT showed that reinforcing amygdala responses to positive autobiographical memories ${ }^{3}$ was superior versus a control neurofeedback intervention ${ }^{4}$. We have developed neurofeedback of self-blame-selective functional connectivity between right superior anterior temporal (AT) and subgenual frontal regions. In remitted MDD, we demonstrated that self-esteem can be increased using this approach in a double-blind RCT. In a recently completed RCT in early treatment-resistant MDD, the majority of patients responded to guilt-related AT-subgenual connectivity neurofeedback. Surprisingly, a self-guided matched psychological intervention tackling self-blame without neurofeedback showed comparable levels of response. Secondary analyses, however, showed that neurofeedback was superior for those patients without anxious distress features.

Conclusions This calls for longer-term studies to reproduce previous results and stratified trials to combine psychological and neurofeedback interventions. As a research tool, neurofeedback uncovers causal relationships between functions and anatomical subdivisions.

\section{REFERENCES}

1. D. E. Linden, et al., PloS one 2012;7:e38115.

2. D. M. A. Mehler, et al., Neuropsychopharmacology, (2018).

3. K. D. Young, et al., PloS one 2014;9:e88785.

4. K. D. Young, et al., Am. J. Psychiatry 2017;174:748-755.

\section{THE EPIDEMIOLOGY AND SYMPTOMOLOGY OF FUNCTIONAL STROKE MIMICS: A SYSTEMATIC REVIEW AND META-ANALYSIS}

${ }^{1}$ Abbeygail Jones, ${ }^{1}$ Nicola O'Connell, ${ }^{2}$ Anthony David. ${ }^{1}$ Institute of Psychiatry, Psychology and Neuroscience, King's College London; ${ }^{2}$ Institute of Mental Health, University College London

\subsection{6/jnnp-2019-BNPA.35}

Aims Reconfiguration of stroke services in England has emphasised fast diagnosis and treatment and subsequently, the proportion of stroke mimic patients entering stroke pathways has been highlighted. Stroke mimic patients may be 'medical mimics', with medical explanations for symptoms, e.g. syncope, seizure, but a proportion of presenting patients have a 